\title{
Implementation and Comparison of Image Enhancement Techniques
}

\author{
Swati Khidse \\ Research Student \\ Government College of Engineering, Station Road, \\ Osmanpura, Aurangabad, Maharashtra, India
}

\begin{abstract}
Image enhancement is the key and important step in digital image processing. If the image is not clear, it cannot be able to perform precisely edge detection, segmentation and other image processing steps. For enhancement of images that are multiresolution, image fusion provides the best output results. Image fusion is the technique of combining relevant information from source images, to get the fused image having most of the information from the source images. This technique can be used in various application areas like aerial images, forensic, flash photography, real life photographs and etc. In this paper, authors discusses the implementation of three categories of image fusion algorithms - basic fusion algorithms, pyramid based algorithms and the basic DWT algorithms and these algorithm are assessed using various objective assessment metrics for image enhancement .These fusion algorithms are compared against the general image enhancement methods for different images with the help of error analysis techniques i.e. Average Difference(AD), Normalized Mean Square Error (NMSE) and the Peak Signal to Noise Ratio (PSNR) and etc.The image fusion methods provide better results than the general image enhancement methods.
\end{abstract}

\section{General Terms}

Digital Image Processing, Image Enhancement

\section{Keywords}

Image Pyramid, Decomposition, Quality Metrics, Principal Component Analysis, Discrete Wavelet Transform, Image Fusion

\section{INTRODUCTION}

Image enhancement is the method for improving perception of information from the images for human viewers and providing better input for the automated image processing techniques. The images which are captured from real life scenario with high dynamic range contain both dark and bright regions. The objective of image enhancement is to modify attributes of the captured image to make it more suitable for further processing. During this process, attributes of the image are modified according to the method applied on that. The choice of attributes and the way they are varied are specific to a given application. [10] [11]

\subsection{Image enhancement techniques}

Image enhancement techniques can be classified into two categories namely -Spatial domain, Frequency domain. [7] [14]

\subsubsection{Spatial Domain}

Spatial domain technique enhances an image by direct manipulation of pixels in an image. [1][7][11]

\author{
Meghana Nagori \\ Assistant Professor CSE Dept. \\ Government College of Engineering, Station Road, \\ Osmanpura, Aurangabad, Maharashtra, India
}

\subsubsection{Image Negative}

The negative of an image can be obtained by using the negative transformation with gray levels in the range $[0, \mathrm{~L}-1]$.

$S=L-1-\gamma$

where $r \& s$ denote the values of the pixels before and after the processing. This method is used for enhancing white or gray detail embedded in the dark regions of an image.

\subsubsection{Log Transform}

The Log transform can be given by $s=c \log (1+r)$

$C$ is a constant. This transformation maps a narrow range of low gray-level values to the input image in a wider range of output levels.

\subsubsection{Power Law Transform}

It can be given by the expression below

$s=C r^{Y} \quad$ where $C$ and $\gamma$ are constants. Value of $\gamma>1$ enhances the contrast of high-value portions of the image at the expense of low-value regions, while reverse for $\gamma<1$. This gives the power-law transform properties similar to both the logarithmic $(\gamma<1)$ and exponential $(\gamma>1)$ transforms. The constant $\mathrm{c}$ performs range scaling.

\subsubsection{Exponential Transformation}

This transformation is inverse of Log transform. It enhances the detail in high-value regions (bright) while decreasing the dynamic range in low-value regions (dark) of the image.

\subsubsection{Gray Level Slicing}

In this method fixed threshold values are taken for highlighting a specific range of gray values in the image. There are two different techniques for implementing this, one is Gray-Level Slicing without Preserving Background and another is Gray-Level Slicing with Background.

\subsubsection{Contrast Enhancement}

The basic objective behind contrast enhancement is to increase the dynamic range of gray levels of the image because of poor illumination, lack of contrast because of certain conditions.

\subsubsection{Logical Operations}

AND and OR operations are used for the masking. The sub images in an image can be selected with the help of these operations, operate on a pixel-by-pixel basis. The pixel of the given input image and the mask are logically ANDed or ORed for obtaining results. Depending on the mask the user can obtain the desired part and skip the undesired part of a particular image as required by his application. 


\subsubsection{Spatial filtering}

Spatial filtering process consists of moving the filter mask from point to point in the given image. Each point in the resultant image is the response of filter, calculated using a predefined relationship. [1][10][11]

\subsubsection{Averaging}

The pixel in the output image is simply the average of the pixels contained in the neighborhood of the filter mask.

\subsubsection{Median}

For each pixel, it is replaced by the median of neighborhood rather than the average.

\subsubsection{Frequency Domain}

Frequency domain image enhancement technique basically works by manipulating the Fourier coefficients. This method describes mathematical functions with respect to the frequency and it operates on Frequency transform i.e. Fourier coefficients discrete wavelet transform (DWT), and discrete cosine transform (DCT). [11]

\subsubsection{Low pass filter}

A filter which passes low frequencies and attenuates the high frequencies is called Low Pass Filter. It is used for smoothening (blurring) the image. Smoothness increases and the problem of ringing reduces from Ideal to Gaussian. There are three different types of filters-Ideal, Butterworth, and Gaussian.

\subsubsection{High pass filter}

A filter which passes high frequencies and attenuates the pass frequencies is called High Pass Filter. It is used for sharpening of the image. Sharpening of the image increases from Ideal to Gaussian. There are three different types of filters-Ideal, Butterworth, and Gaussian.

\subsection{Image enhancement with image fusion}

If there are multiresolution images, high dynamic range images that are captured from real world applications, then one image alone cannot provide all the relevant information of the image, if the above methods are used for enhancement. It is not always suitable to use them. Image fusion provides a better way to deal with these kinds of images. It fuses all the relevant information from the source images into the output image.

\section{IMAGE FUSION ALGORITHMS}

The image fusion algorithms can be classified into three different categories- Simple Image Fusion, Pyramid Decomposition based fusion, and Discrete Wavelet transform based fusion. [5] [6] [7] [11] [13]

\subsection{Simple Fusion Algorithms}

The simple fusion algorithm works on basic operation like pixel selection, addition, subtraction or averaging.

\subsubsection{Average}

Every corresponding pixel in the input images is average and resultant image is generated.

\subsubsection{Select Maximum/Minimum}

For every corresponding pixel in the input images, the pixel with maximum/minimum intensity value is selected, respectively, and is replaced in the resultant pixel of the fused image.

\subsubsection{Principal Component Analysis(PCA)}

PCA reduces the multidimensional data sets to the lower dimensions data sets for analysis. PCA algorithm for image fusion is computed from the input image matrices with the help of computing covariance matrix.

\subsection{Pyramid Fusion Algorithms}

Image pyramid [5] [6] [7] [12] [13] consists of a set of low pass or band pass copies of an image. Each copy represent the pattern information of a different scale. At every level in pyramid transform, the pyramid would be half the size of the pyramid in the previous level and the higher levels will concentrate upon the lower spatial frequencies. It consists of three major phases.

\subsubsection{Decomposition}

Decomposition- It is a process where a pyramid is generated successively, at each level. The depth of fusion is pre decided. It consists of following steps

1. Low pass filtering- Input images are convolved/filtered with pre defined filter.

2. Formation of the pyramid- Filtered/convolved input images using Burt's method.

3. Input images are decimated to half their size.

\subsubsection{Initial image for recomposition}

After decomposition process the input images are merged. This resultant image matrix will be the initial input to the recomposition process. Then finally the decimated input pair of images is selected upon either by averaging, or selecting first or second decimated input image.

\subsubsection{Recomposition}

The resultant fused image is developed from the pyramids formed at each level of decomposition. The following steps are performed ' $n$ ' number of times as in the decomposition process. [5]

1. Input image is undecimated to the level of recomposition.

2. The undecimated matrix is filtered with the transpose of the filter vector.

3. The filtered matrix is then merged (pixel intensity value addition), with the pyramid formed at the respective level of decomposition.

4. The new image matrix would now act as the input to the next level. The merged image at the final level will be the resultant fused image.

The different pyramid algorithms are

\subsubsection{Filter Subtract Decimate Pyramid(FSD)}

Decomposition phase- Use $\mathrm{W}$ for Low pass filtering. $W=\left[\frac{1}{16} * \frac{4}{16}, \frac{6}{16}, \frac{4}{16} * \frac{1}{16}\right]$

After this subtract the low pass filtered input images and then form the pyramid. Then lastly decimate the input image matrices, by halving the number of rows and columns.

Recomposition phase - Undecimate the image matrix. (duplicate every row and column). After this perform Low pass filtering with $2 * \mathrm{~W}$ followed by matrix addition with the pyramid formed in that corresponding level. 


\subsubsection{Laplacian Pyramid(LP)}

In this pyramid method additional low pass filtering is performed with $2 * \mathrm{~W}$. All the remaining steps are similar as in FSD pyramid. [2]

\subsubsection{Ratio Pyramid(RP)}

Ratio pyramid method is identical to FSD except, in the decomposition phase, after low pass filtering of the images; the pixel wise ratio is calculated instead of subtraction.

\subsubsection{Gradient Pyramid(GP)}

Decomposition process

1. Consider two low pass filters

$$
\mathrm{W}=\left[\frac{1}{16}, \frac{4}{16}, \frac{6}{16}, \frac{4}{16}, \frac{1}{16}\right] \text { and } \mathrm{V}=\left[\frac{1}{4}, \frac{2}{4}, \frac{1}{4}\right]
$$

2. Four directional filters are applied over input image matrices in addition to low pass filters.

Horizontal filter $\left[\begin{array}{rrr}0 & 0 & 0 \\ 1 & -2 & 1 \\ 0 & 0 & 0\end{array}\right]$

Vertical filter $\left[\begin{array}{rrr}0 & 1 & 0 \\ 0 & -2 & 0 \\ 0 & 1 & 0\end{array}\right]$

Diagonal filter $\left[\begin{array}{crc}0 & 0 & 0.5 \\ 0 & -1 & 0 \\ 0.5 & 0 & 0\end{array}\right]$

Diagonal filter $\left[\begin{array}{crr}0.5 & 0 & 0 \\ 0 & -1 & 0 \\ 0 & 0 & 0.5\end{array}\right]$

3. Remaining steps are as in the FSD pyramid method.

\subsubsection{Morphological Pyramid (MP)}

Decomposition phase:

1. Two levels of filtering - Image opening and image closing are performed on input image matrices. Image opening is a combination of image erosion followed by image dilation on the other hand image closing is a combination of image dilation followed by image erosion.

2. Remaining steps are similar to that of FSD pyramid.

Recomposition phase: Image dilation is performed over the matrix instead of low pass filter; remaining steps are similar to the FSD.

\subsection{Discrete Wavelet Transform}

The DWT [5] [6] [7] is used when the images are to be processed at multiple resolutions. The Fourier transform of the digital image reveals only its frequency attributes on the other hand DWT reveals image's spatial and frequency characteristics. The DWT of a signal $\mathrm{x}$, can be calculated by passing it through a series of filters. The samples are firstly passed through a low pass filter with impulse response $g$.

$Y(n)=(x * g)[n]=\sum x[k] g[n-k]$

It is decomposed simultaneously with a high-pass filter $h$. The outputs are detail coefficients and approximation coefficients.

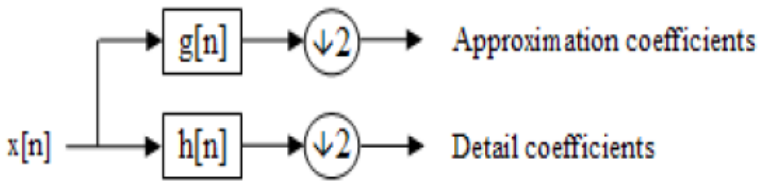

Figure 1: Decomposition of signal

The decomposition process is repeated further to increase the frequency resolution and the approximation coefficients decomposed with high and low pass filters. Figure 2 represents the flowchart of the wavelet image fusion with decomposition and reconstruction phases.

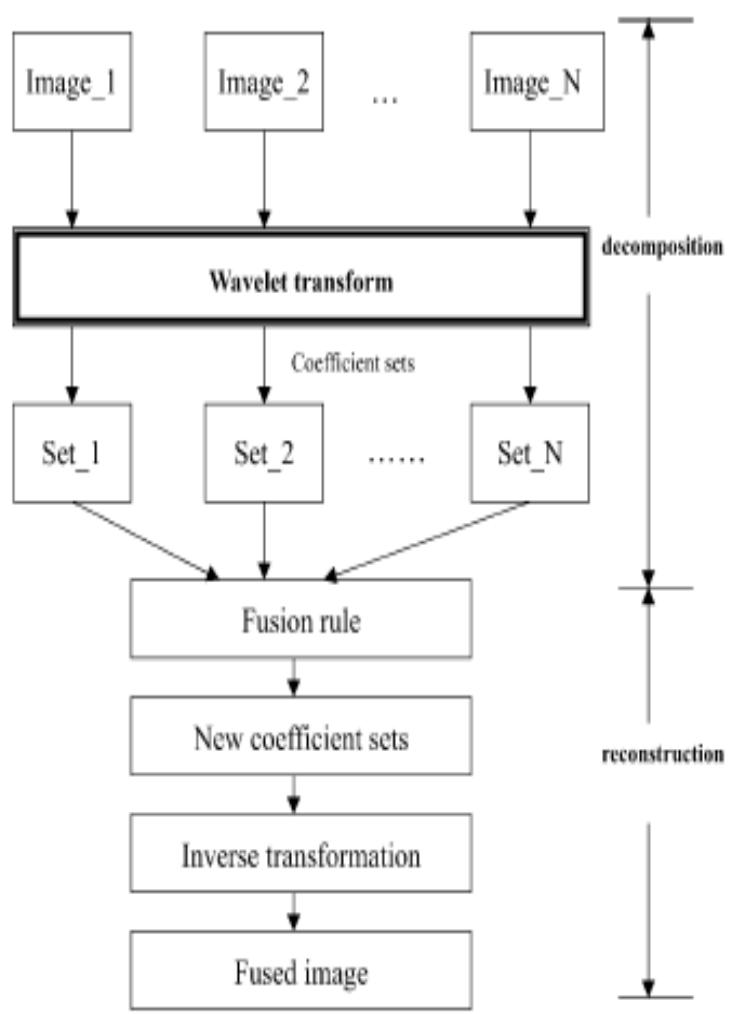

Figure 2: The flowchart of wavelet image fusion

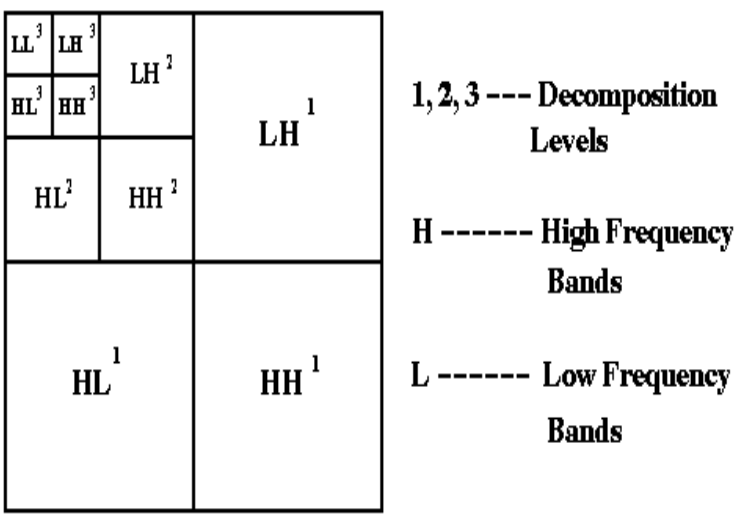

Figure 3: Structure of 2-D DWT with 3 decomposition levels 


\subsubsection{DWT with Haar based fusion}

The Haar wavelet $\psi(t)$ and its scaling function $\varphi(t)$ can be described as:

$$
\Psi(t)=\left\{\begin{array}{cl}
1 & 0 \leq t \leq \frac{1}{2} \\
-1 & \frac{1}{2} \leq t \leq 1 \\
0 & \text { Otherwise }
\end{array}\right.
$$

When filters are, applied on the input images matrices, it would produce 4 resultant matrices. The fourth matrix would consist of all the high frequencies, and it will act as the input matrix for the next level of the decomposition process. The other three matrices consist of the low frequencies, used to produce 3 pyramids at each level and also used for recomposition process. [5] [7] [15]

\subsubsection{DWT with DBSS (2,2) based fusion}

The [5] [16] Daubechies wavelets are a family of orthogonal wavelets defining a DWT. They are generally characterized by a maximal number of vanishing moments for some support; there is also a scaling function which will generate an orthogonal multiresolution analysis. Generally the vanishing moments are chosen to have the highest number $\mathrm{A}$ for given support width $\mathrm{N}=2 \mathrm{~A}$.

\section{PERFORMANCE EVALUTION}

The objective assessment of the [8] [11] various image fusion algorithms can be performed with the following metrics.

\subsection{Information theory based metrics:}

3.1.1 Normalized Mutual Information $\left(Q_{\mathrm{MI}}\right)$ : It represents mutual dependence of two variables. Mutual information is represented by MI (A, F), entropy with $\mathrm{H}(\mathrm{A})$.

$Q_{\mathrm{MN}}=2\left[\frac{M P\left(A_{x} F\right)}{H(A)+H(F)}+\frac{M\left(B_{v} F\right)}{H(B)+H(F)}\right]$

\subsubsection{Fusion Metric-Based on Tsallis Entropy}

$\left(Q_{T E}\right)$ : Tsallis entropy represents the divergence measure of degree of dependence between two variables.

$Q_{T E}=\frac{I^{Q}\left(A_{v} F\right)+I^{Q}\left(B_{v} F\right)}{H^{Q}(A)+H^{Q}(B)-I^{Q}\left(A_{v} B\right)}$

3.2 Image feature based metrics: Assessment is implemented by measuring how the features are transferred from the input images to the fused one.

3.2.1 Image Fusion Metric-Based on Spatial Frequency $\left(Q_{S F}\right)$ : Spatial Frequency is used to measure the activity level of an image $\mathrm{I}(\mathrm{x}, \mathrm{y})$.

$S F=\sqrt{(R F)^{2}+(C F)^{2}+(M D F)^{2}+(S D F)^{2}}$,

$Q_{S P}=\left(S F_{F}-S F_{R}\right) / S F_{R}$

$\mathrm{RF}, \mathrm{CF}, \mathrm{SDF}$, and MDF are the four first-order gradients along the four directions.
3.3 Image structural similarity based

metrics: A structural similarity index measure (SSIM) for images $\mathrm{A}$ and $\mathrm{B}$ defined as

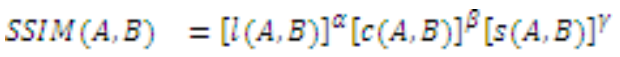

$$
\begin{aligned}
& =\left(\frac{2_{\mu_{A} \mu_{B}}+C_{1}}{\mu_{A}^{2}+\mu_{B}^{2}+C_{1}}\right)^{\mathscr{D}}\left(\frac{2_{\sigma_{\alpha} \sigma_{B}}+C_{2}}{\sigma_{A}^{2}+\sigma_{B}^{2}+C_{2}}\right)^{\beta}\left(\frac{\sigma_{A B}+C_{2}}{\sigma_{A}+\sigma_{B}+C_{I}}\right)^{F}
\end{aligned}
$$

In this $\mu_{A}$ and $\mu_{B}$ are the average values of images A (i, j) and $\mathrm{B}(\mathrm{i}, \mathrm{j}), \sigma_{A}, \sigma_{B}$, and $\sigma_{A B}$ are the variance and covariance. The 1 (A, B), c (A, B) and s (A, B) are the luminance, contrast, and correlation components, resp.

\section{ERROR ANALYSIS TECHNIQUES}

Various Error analysis techniques [5] [9] [11] can be used for comparisons:

\subsection{Normalized Mean Square Error}

Method (NMSE): The NMSE compares the mean of a series against the predicted values. If the NMSE value is greater than 1 , then the predictions are going worse than the series mean and vice versa. The following formula is used to calculate NMSE:

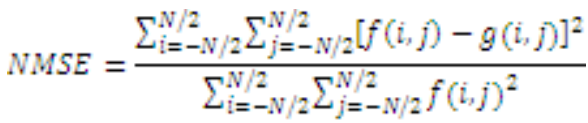

Where $f\left(\mathbb{i}_{0} j\right)$ is the original image with size $\mathrm{N} * \mathrm{~N}$, and $g\left(\hat{i}_{0} j\right)$ is the filtered image with size $\mathrm{N}^{*} \mathrm{~N}$.

\subsection{Peak Signal to Noise Ratio (PSNR):}

This basically represents the ratio of the highest possible value of the digital image data to the error obtained in the digital image data, where MSE is Mean Square Error.

$$
P S N R=10 \times \log _{10}\left(\frac{\text { peak }^{2}}{M S E}\right)
$$

4.3 Normalized Absolute Error (NAE): NAE is the average of the absolute difference between the originally and the enhanced image divided by the original image. The large the value of NAE the poor is the image.

$N A E=\frac{\sum_{i=1}^{W} \sum_{j=1}^{W}\left(\left\|A_{i j}-B_{i j}\right\|\right)}{\sum_{i=1}^{W} \sum_{j=1}\left(A_{i j}\right)}$

4.4 Maximum Difference (MD): It represents the maximum difference between two images.

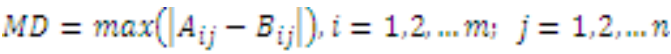

4.5 Average Difference (AD): This measure shows the average difference between the pixel values given by

$A D=\frac{1}{m m} \sum_{i=1}^{3 m} \sum_{j=1}^{n n}\left(A_{i j}-B_{i j i}\right)$ 
4.6 Normalized Cross-Correlation (NCC): Normalized cross correlation are used to find out similarities between fused image and registered image is given by

NCC $=\frac{\sum_{i=1}^{W} \sum_{j=1}^{W}\left(A_{i j} * B_{i j}\right)}{\sum_{i=1}^{W} \sum_{j=1}^{W}\left(A_{i j}\right)^{2}}$

4.7 Structural Content (SC): It is correlation based measure for the original and enhanced image.

$S C=\frac{\sum_{i=1}^{M} \sum_{j=1}^{W}\left(A_{i j}\right)^{2}}{\sum_{i=1}^{M} \sum_{j=1}^{M}\left(B_{i j}\right)^{2}}$

\section{IMPLEMENTATION AND RESULTS}

According to the information of Table 1 [11] the image enhancement is classified into two domains spatial domains and frequency domain in both techniques. Firstly the image is enhanced using the general image enhancement techniques and one of the best methods is selected from the error analysis techniques. For the corresponding images, best method among all and the metric values are given in Table 2. But this method does not provide good results in case of the multiresolution images or HDR images in which a single image is not able to provide all the relevant information from the source. In this scenario image fusion provide more good results than the previous method. Table 3 provides the quality metrics values for different images, in this the method which is better than all is given along with their values. Table 4 provides the information regarding image fusion algorithms. For any particular multiresolution images as the input images all the

Table 1: Image enhancement \& fusion methods

\begin{tabular}{|c|c|c|c|}
\hline Sr.no & $\begin{array}{l}\text { Type of } \\
\text { images }\end{array}$ & Image enhancement & Image fusion \\
\hline [1] & $\begin{array}{l}\text { Satellite } \\
\text { image } \\
\text { And etc. }\end{array}$ & 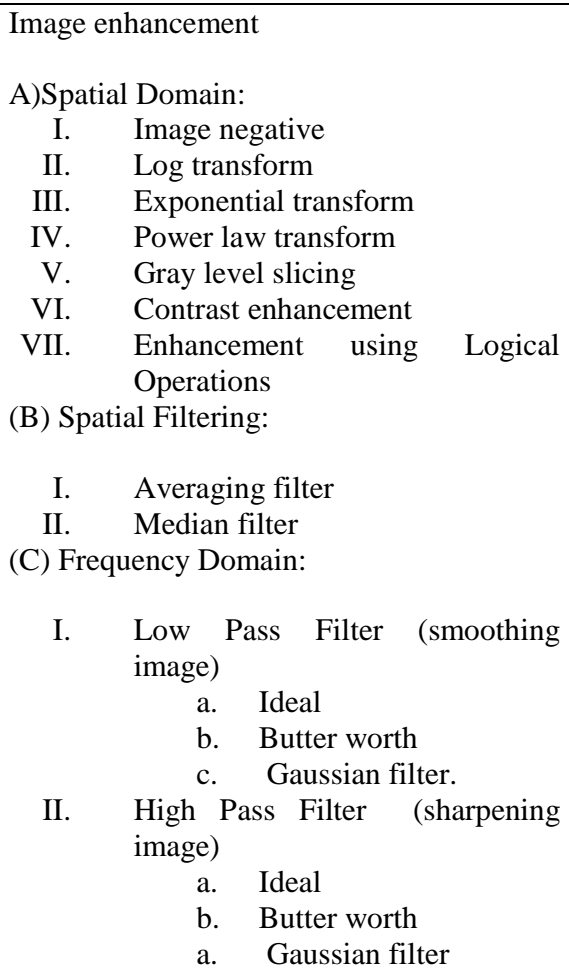 & 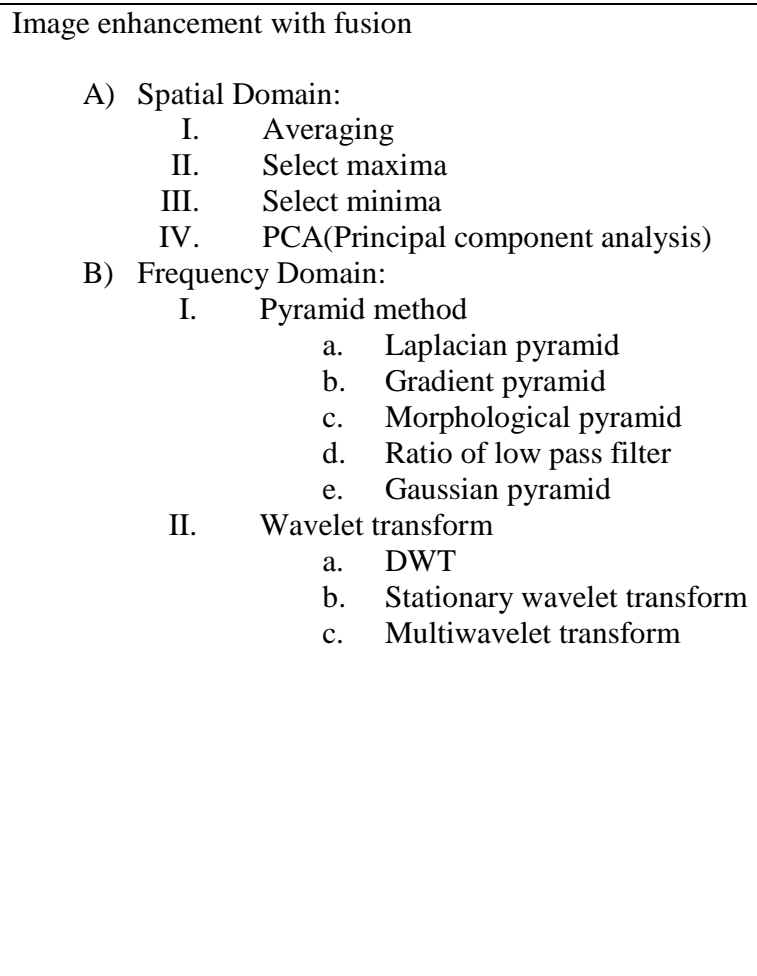 \\
\hline
\end{tabular}

Table 2: Quality metrics values using image enhancement methods

\begin{tabular}{|c|c|l|c|c|c|c|c|c|c|}
\hline $\begin{array}{c}\text { Sr. } \\
\text { No. }\end{array}$ & Image & $\begin{array}{c}\text { Method(Best } \\
\text { Results) }\end{array}$ & AD & MD & NMSE & NAE & NCC & PSNR & SC \\
\hline 1 & & $\begin{array}{l}\text { Contrast } \\
\text { enhancement }\end{array}$ & -0.6226 & 1 & 0.0007 & 0.0367 & 1.0258 & 39.7967 & 0.9501 \\
\hline
\end{tabular}




\begin{tabular}{|c|c|c|c|c|c|c|c|c|}
\hline 2 & $\begin{array}{c}\text { Gaussian Low } \\
\text { pass filter }\end{array}$ & 0.0430 & 52.2277 & 0.0006 & 0.0153 & 0.9979 & 38.7813 & 1.0034 \\
& & & & & & & & \\
\end{tabular}

Table 3: Quality metrics values using image enhancement

\begin{tabular}{|c|c|c|c|c|c|c|c|c|}
\hline Sr. No. & $\begin{array}{c}\text { Method(Best } \\
\text { Results) }\end{array}$ & AD & MD & NMSE & NAE & NCC & PSNR & SC \\
\hline 1 & Contrast & -23.643 & 10 & 0.2517 & 0.4643 & 1.4529 & 17.9255 & 0.4634 \\
\hline 2 & Power Law & 51.534 & 254 & 1.9887 & 1.7925 & 0.0056 & 10.6196 & 28003 \\
\hline 3 & Exponential & 25.6424 & 33.1907 & 0.0292 & 0.1788 & 0.8497 & 19.7555 & 1.3722 \\
& & & & & & & & \\
\hline
\end{tabular}

Table 4: Quality metrics values \& objective assessment of image fusion algorithm

\begin{tabular}{|c|c|c|c|c|c|c|c|c|c|c|c|c|}
\hline $\begin{array}{l}\text { Sr. } \\
\text { No. }\end{array}$ & $\begin{array}{c}\text { Fusion } \\
\text { (Best } \\
\text { Results) }\end{array}$ & AD & MD & NMSE & NAE & NCC & PSNR & SC & $Q_{\text {NI }}$ & $Q_{T E}$ & $Q_{s 5}$ & SSIM \\
\hline 1 & $\begin{array}{l}\text { Gradient } \\
\text { Pyramid }\end{array}$ & 2.9615 & 137.414 & 0.1277 & 0.3547 & 0.828 & 19.82 & 1.275 & 0.171 & -0.397 & -0.091 & 0.662 \\
\hline 2 & DWT & -86.646 & 15.0936 & 1.4632 & 1.6704 & 1.724 & 8.917 & 0.255 & 0.524 & 4.719 & -0.006 & 0.458 \\
\hline 3 & DWT & -0.7097 & 133.02 & 0.0021 & 0.0247 & 1.004 & 31.047 & 0.988 & 0.950 & 18.01 & 0.432 & 0.885 \\
\hline
\end{tabular}

Table 5: Input and output images of various techniques

\begin{tabular}{|c|c|c|c|c|}
\hline Sr. No. & Image1 & Image2 & Image Enhancement & Image Fusion \\
\hline 1 & Footprint1 & Footprint2 & Output1 & 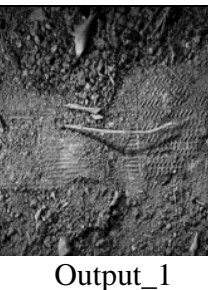 \\
\hline
\end{tabular}




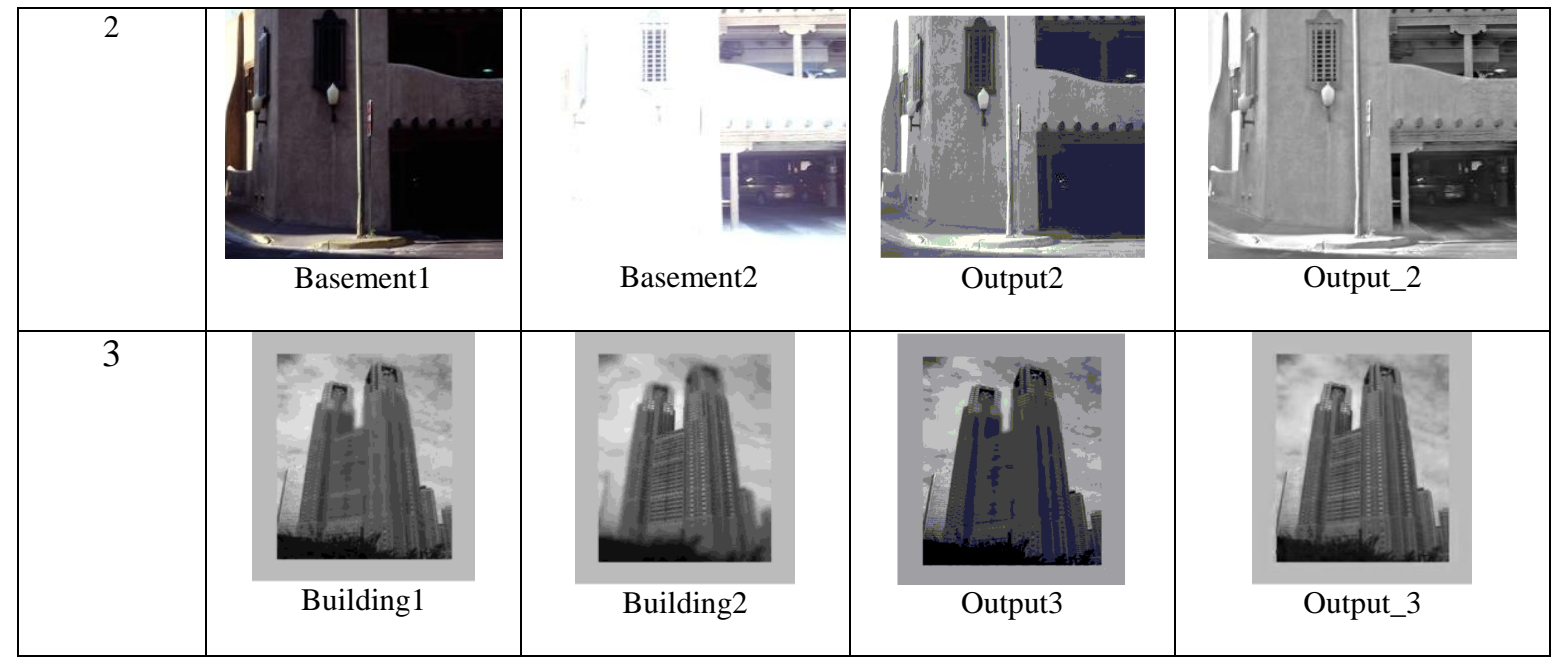

methods of spatial domain and frequency domain of Table 1 are performed along with all the performance evaluation methods i.e. Objective assessment is performed over input images- Information theory based metrics, Image feature based metrics, Image structural similarity based metrics. Error Analysis Techniques is also performed for each image fusion method. Based on this computation values one of the best techniques is selected from all methods. Table 5 represents the information regarding the input images and the output images of both the techniques i.e. image enhancement general methods and the image fusion.

\section{CONCLUSION}

Image is the main source of acquiring information from the real world. It is not always possible to extract all the information from one source image; to deal with this problem image fusion provides better results than its counterpart. The values obtained from the image metrics - NMSE, PSNR, SC, $\mathrm{NCC}, \mathrm{AD}, \mathrm{MD}$, and NAE were used to assess the best enhancement techniques in terms of the quality of the output image. The fusion algorithms are also objectively assessed using various metrics. Generally DWT based fusion techniques provides good quality fused images. In image fusion two source images are used for fusing, it can be modified with more than two source images so that the resultant image will be more efficient. This technique can be used in videos also. Image Registration/ Image Alignment can be incorporated in Image fusion method in which the images from different sources will be aligned in same angle, which will enhance the efficiency of the resultant output image. Multiwavelet fusion can also be implemented for improving enhancement of images.

\section{ACKNOWLEDGEMENTS}

The authors express gratitude to Principal, Head of Department (CSE) Prof. V. P. Kshirsagar, Government College of Engineering, Station Road, Aurangabad, Maharashtra India. They also express their sincere thanks to all the faculty members of CSE Department Government College of Engineering, Station Road, Osmanpura, Aurangabad, Maharashtra, India for their constant support and enthusiasm.

\section{REFERENCES}

[1] Atul Bansal, Rochak Bajpai, J. P. Saini, "Simulation of Image Enhancement Techniques Using Matlab", IEEE Computer Society 2007.
[2] N. Indhumadhi, g. Padmavathi, "Enhanced Image Fusion Algorithm Using Laplacian Pyramid and Spatial frequency Based Wavelet Algorithm", International Journal of Soft Computing and Engineering (IJSCE) ISSN: 2231-2307, Volume-1, Issue-5, November 2011, pp. 298-302.

[3] Raman Maini and Himanshu Aggarwal," A Comprehensive Review of Image Enhancement Techniques", journal of computing, volume 2, issue 3, march 2010, ISSN 2151-9617, pp. 8-12.

[4] X. Fang, J. Liu, W. Gu, Y. Tang, “ A Method to Improve the Image Enhancement Result based on Image Fusion" 978-1-61284-774-0/11 (C2011 IEEE.

[5] Shivsubramani Krishnamoorthy, K. P. Soman, "Implementation and Comparative Study of Image Fusion Algorithms", IJCA 2010, pp.25-35.

[6] C.Y. Wen, J.K. Chen, "Multi-resolution image fusion technique and its application to forensic science", 2004 Elsevier Ireland Ltd, pp.217-231.

[7] Rafael, C. Gonzalez and R. E. Woods, Digital image processing: 2nd edition, Prentice Hall, 2002.

[8] Zheng Liu, Erik Blasch, Zhiyun Xue, Jiying Zhao, Robert Laganie, Wei Wu, "Objective Assessment of Multiresolution image fusion Algorithms for Context Enhancement in Night Vision: A Comparative Study", IEEE transactions on pattern analysis and machine intelligence, vol. 34, no. 1, January 2012, pp.95-98.

[9] Moustafa Abdel Aziem Moustaf, "Quntitative and qualitative evaluations of image enhancement techniques", IEEE 2004, pp.665-666.

[10] Er. Nancy, Er. Sumandeep Kaur, “Comparative Analysis and Implementation of Image Enhancement Techniques Using MATLAB”, IJCSMC, Vol. 2, Issue. 4, April 2013, pg.138- 145 .

[11] Swati Khidse, Meghana Nagori,"A Comparative Study of Image Enhancement Techniques", International Journal of Computer Applications (0975 - 8887) Volume 81 - No 15, November 2013, pp.28-32.

[12] F. Sadjadi, "Comparative Image Fusion Analysais", IEEE Computer Society Conference on Computer Vision and Pattern Recognition, Volume 3, Issue , 20-26 June 2005 Page(s): $8-8$ 
[13] Anjali Malviya, S. G. Bhirud, "Image Fusion of Digital Images", International Journal of Recent Trends in Engineering, Vol 2, No. 3, November 2009, pp.146-147.

[14] A. K. Jain, "Fundamentals of Digital Image Processing", Prentice Hall of India, First Edition,1989.
[15] "Haar Wavelet"
http://en.wikipedia.org/wiki/Haar_wavelet

[16] "Daubechies

Wavelets", http://en.wikipedia.org/wiki/Daubechies_wavelet 